\title{
ROOTS OF UNITY AND THE CHARACTER VARIETY OF A KNOT COMPLEMENT
}

\author{
D. COOPER and D. D. LONG
}

(Received 15 April 1992)

Communicated by J. H. Rubinstein

\begin{abstract}
Using elementary methods we give a new proof of a result concerning the special form of the character of the bounded peripheral element which arises at an end of a curve component of the character variety of a knot complement.
\end{abstract}

1991 Mathematics subject classification (Amer. Math. Soc.): 57 M 25, 57 N 10.

\section{Introduction}

In [3] the following theorem is proved:

THEOREM 1.1. Suppose that $\rho_{n}$ is a sequence of representations of the fundamental group of a knot which blows up on the boundary torus $T$, and which converge to a simplicial action on a tree. Suppose that there is an essential simple closed curve $C$ on $T$ whose trace remains bounded. Then $\lim _{m \rightarrow \infty} \operatorname{tr}\left(\rho_{m}(C)\right)=\lambda+1 / \lambda$ where $\lambda^{n}=1$ whenever there is a component $S$ of a reduced surface associated to the degeneration so that $S$ has $n$ boundary components.

Precise definitions of the terms will be given below, but the rough description is as follows. If one has a curve of characters of representations of a manifold

(c) 1993 Australian Mathematical Society 0263-6115/93 $\$ A 2.00+0.00$

This paper was supported in part by the Alfred P. Sloan and National Science Foundations 
with a single torus boundary component, then the method of [5] for producing boundary slopes is to go to some end of the character variety. Two things can happen on the boundary torus when one does this; either all the characters remain bounded and the surface produced from the resulting splitting can be chosen to be closed, or there is a particular simple closed curve whose character remains bounded. We shall focus on this latter behaviour. This simple closed curve gives the boundary slope and a natural question to ask is what the value of the character of the closed curve at the ideal point is. The point of the theorem is that the character has a special form and that some information about this form is carried by the topology of a splitting surface coming from the degeneration.

As an aside, we note that the theorem shows that for a two-bridge knot only the numbers \pm 1 can occur, since it is known ([6]) that the essential surfaces in such knot complements have either one or two boundary components. It is also known that other values are possible - the untwisted double of the trefoil contains an ideal point where the bounded character takes on the value $\omega+\omega^{-1}$ where $\omega$ is an eleventh root of unity. But other than this, little is known. For example, it still seems to be an open question whether a nontrivial root of unity can arise in this way in the character variety of a hyperbolic knot.

In this paper we shall give a new proof of Theorem 1.1. In fact it is a geometric version of one of the proofs of [3], but the fact that it avoids both algebraic $K$-theory and algebraic geometry and provides a somewhat new perspective should hopefully yield some new insights.

The point of view of this proof is that the action on a tree produced by the techniques of [5] is approximated in a geometrical sense by the action of the representations $\rho_{m}$ for $m$ large. This is the idea used in [1] and also [2].

\section{Main results}

LEMMA 2.1. Given $L>0$ and $n>0$ there is a constant $K_{n}>0$ such that for any set of matrices $A_{1}, A_{2}, \ldots, A_{n} \in \mathrm{SL}_{2}(\mathrm{C})$ with $\left|\operatorname{tr}\left(A_{i} A_{j}\right)\right|<L$, for all $1 \leq i, j \leq n$ then there is a point $x \in \mathbf{H}^{3}$ which is moved a distance of at most $K_{n}$ by $A_{i}$ for every $i$.

PROOF. The proof is by induction on $n$. For $n=1$, the result follows from the relationship between trace and translation length. For $n=2$, suppose that we are given a pair of matrices $A, B$ in $\mathrm{SL}_{2}(\mathrm{C})$. The proof proceeds by showing that we can simultaneously conjugate $A$ and $B$ so that they are in the compact subset $\Omega$ of $\mathrm{SL}_{2}(\mathrm{C})$, where: 


$$
\Omega=\left\{\left(\begin{array}{ll}
a & b \\
c & d
\end{array}\right) \in \mathrm{SL}_{2}(\mathbf{C}):|a|,|b|,|c|,|d| \leq 2 L+2\right\}
$$

If $A$ and $B$ have a common fixed point on the sphere at infinity, then we may perform a simultaneous conjugacy on them to put the common fixed point at infinity in the upper half space, then:

$$
A=\left(\begin{array}{cc}
a & c \\
0 & 1 / a
\end{array}\right), \quad B=\left(\begin{array}{cc}
b & d \\
0 & 1 / b
\end{array}\right) .
$$

Furthermore, by conjugating by a diagonal matrix we may ensure that $|c|,|d| \leq$ 1. The hypothesis implies that $1 /(L+1)<|a|^{2},|b|^{2}<(L+1)$ so that $A, B$ lie in $\Omega$.

If $A$ and $B$ do not have a common fixed point then there is a point $z$ on the sphere at infinity which is fixed by $A^{-1} B$. By means of a conjugacy we can arrange that $z=0$ and that $B z=\infty$. Thus $A z=\infty$ also and thus $A ; B$ are conjugate to:

$$
A=\left(\begin{array}{cc}
a & 1 \\
-1 & 0
\end{array}\right), \quad B=\left(\begin{array}{cc}
b & c \\
-1 / c & 0
\end{array}\right)
$$

The hypothesis implies that $|a|^{2},|b|^{2}<L+2$. Observe that $\operatorname{tr}(A B)=a b-$ $c-(1 / c)$, thus $|c+(1 / c)|<2 L+2$, and so $A$ and $B$ lie in $\Omega$.

Given a point $x$ in $\mathbf{H}^{3}$ the function which assigns to a pair of matrices $(A, B)$ the maximum of the hyperbolic distance of $x$ from $A x$ and from $B x$ is continuous, and therefore bounded on $\Omega \times \Omega$. Since the existence of a point $x$ satisfying the conclusion is invariant under conjugacy, the result for $n=2$ follows.

Suppose inductively that the result is true for any set of $(n-1)$ matrices with a constant $K_{n-1}$. Given a set of $n \geq 3$ matrices satisfying the hypothesis, let $x_{i}$ be a point moved a distance at most $K_{n-1}$ by the matrices $\left\{A_{j} \mid 1 \leq j \leq n, j \neq i\right\}$. Define $C_{i}$ to be the convex hull of the finite set $\left\{x_{j} \mid j \neq i\right\}$ and consider the goedesic triangle $T$ with vertices $\left\{x_{1}, x_{2}, x_{3}\right\}$. The radius of the largest circle which may be inscribed in a geodesic triangle is $2 \ln [(1+\sqrt{5}) / 2]$ thus there is a point $y$ which lies within this distance of each side of $T$. Each $C_{i}$ contains at least two vertices of $T$, and therefore at least one edge of $T$. Therefore $y$ lies within a distance of $2 \ln [(1+\sqrt{5}) / 2]$ of $C_{i}$. The vertices of $C_{i}$ are moved at most a distance of $K_{n-1}$ by the matrix $A_{i}$, therefore every point of $C_{i}$ is moved at most a distance $K_{n-1}$ by $A_{i}$. This uses the fact that the distance between a 
point and its image under an isometry, is a convex function. Thus the distance of $y$ from $A_{i} y$ is at most $K_{n-1}+4 \ln [(1+\sqrt{5}) / 2]$.

COROLlaRY 2.1. Suppose that $G$ is a finitely generated group and that we are given that $\rho_{n}: G \longrightarrow \mathrm{SL}_{2}(\mathrm{C})$ is a sequence of representations which have characters $\chi_{n}=$ trace $\circ \rho_{n}$ which converge weakly to a function $\chi$. Then there is a subsequence $\rho_{n_{i}}$ and matrices $A_{i} \in \mathrm{SL}_{2}(\mathrm{C})$ such that $A_{i} \cdot \rho_{n_{i}} \cdot A_{i}^{-1} \rightarrow \rho$ and trace $\circ \rho=\chi$.

ProOF. Choose a finite set of elements $\left\{\alpha_{1}, \alpha_{2}, \cdots, \alpha_{p}\right\}$ which generate $G$, then by Lemma 2.1 we have that for $n$ sufficiently large there is $x_{n}$ in $\mathbf{H}^{3}$ which is moved a distance at most $K_{p}$ by $\rho \alpha_{i}$ for $i=1,2, \cdots, p$. After conjugating each $\rho_{n}$, we may arrange that $x_{n}=x$ for every $n$. The subset $\Omega$ of $\mathrm{SL}_{2}(\mathbf{C})$ consisting of elements which move $x$ a distance of at most $K_{p}$ is compact. Thus there is a subsequence as claimed.

The set $X\left(G, \mathrm{SL}_{2}(C)\right)$ of characters of representations of a group $G$ into $\mathrm{SL}_{2}(C)$ is given the weak topology. This coincides with the topology induced by an embedding of $X\left(G, \mathrm{SL}_{2}(\mathbf{C})\right)$ into a finite dimensional Euclidean space given by using the traces of a (large enough) finite set of elements of $G$. If $G$ is finitely generatated it follows from Corollary 2.1 that $X\left(G, \mathrm{SL}_{2}(\mathrm{C})\right)$ is a closed subset of Euclidean space. (See [5].)

LEMMA 2.2. Suppose that $G$ is a finitely generated group and $\rho_{n}: G \longrightarrow$ $\mathrm{SL}_{2}(\mathbf{C})$ is a sequence of representations with the property that for every $\alpha \in G$, $\operatorname{tr}\left(\rho_{n} \alpha\right) \rightarrow \pm 2$ as $n \rightarrow \infty$. Then after changing each $\rho_{n}$ by a suitable conjugacy, a subsequence of $\left\{\rho_{n}\right\}$ converges to an abelian representation.

PROOF. By Corollary 2.1, we can conjugate a subsequence of the $\rho_{n}$ so that this subsequence converges to a representation $\rho$ for which $\operatorname{tr}(\rho \alpha)= \pm 2$ for every $\alpha$ in $G$. The image of $\rho$ consists entirely of parabolic elements and $\{ \pm I\}$. If two of these parabolics have distinct fixed points, then a large power of one times a large power of the other is hyperbolic, which contradict the hypothesis. Thus $\rho$ is reducible, and so can be conjugated to be upper triangular. Now a sequence of conjugacies by suitable diagonal matrices makes $\rho$ converge to a diagonal representation.

We now study degenerations of knot complements. Let $M$ be the complement of a knot and $\rho_{n}: \pi_{1}(M) \longrightarrow \mathrm{SL}_{2}(\mathbf{C})$ be a sequence of representations. We 
say that this sequence blows up if there is an element $\alpha \in \pi_{1}(M)$ such that $\operatorname{trace}\left(\rho_{n} \alpha\right) \rightarrow \infty$. We assume that the projectivized length functions which they determine converge to some projectivized length function and further, that all these representations lie on a curve in the representation variety. The consequence of this assumption is that the limiting projectivized length function comes from an action of $\pi_{1}(M)$ on a simplicial tree $\Gamma$ rather than an R-tree.

We shall assume that $\pi_{1}(M)$ acts on $\Gamma$ without inversions and that if an edge $e$ in $\Gamma$ is incident to a vertex $v$ then $\operatorname{stab}(e)$ is contained in but not equal to stab $(v)$. Let $e$ be an edge of $\Gamma$; we construct a properly embedded surface $S$ in $M$ from $e$ as follows. Let $\tilde{M}$ be the universal cover of $M$ and choose an equivariant map

$$
f: \tilde{M} \longrightarrow \Gamma
$$

Make $f$ transverse to the midpoint of $e$. Then $\tilde{S}=f^{-1}(e)$ is a properly embedded 2 -sided surface in $\tilde{M}$, possibly not connected. After performing compressions on $\tilde{S}$ by equivariantly homotoping $f$ we may assume that all components of $\tilde{S}$ are planes. We assume that the action of $\pi_{1}(M)$ on $\Gamma$ has no common fixed point. Let $F$ be a component of $S$ which separates $M$ into two components $M_{+}$and $M_{-}$. Then:

$$
\pi_{1}(M)=\pi_{1}\left(M_{+}\right) *_{\pi_{1}(F)} \pi_{1}\left(M_{-}\right) .
$$

For some choice of $F$ this decomposition is non-trivial. We have that $\pi_{1}(F)$ is contained in $\operatorname{stab}(e)$ and there are finite collections of vertices $v_{i}^{ \pm}$of $\Gamma$ with $\pi_{1}\left(M_{+}\right)$contained in the group generated by the union of the $\operatorname{stab}\left(v_{i}^{+}\right)$, and similarly for the minus sign.

PROPOSITION 2.1. With the above assumptions, each $\rho_{n}$ may be replaced by a conjugate so that there is a subsequence of $\rho_{n} \mid \operatorname{stab}(e)$ which converges to an abelian representation.

PROOF. If $\gamma \in \pi_{1}(M)$ has the property that trace $\left(\rho_{n}(\gamma)\right)$ is bounded as $n \rightarrow$ $\infty$, we will say that $\gamma$ remains bounded. By [4], $\gamma$ remains bounded if and only if $\gamma$ stabilizes some vertex of $\Gamma$.

We apply Corollary 2.1 to the sequence of representations $\rho_{n} \mid \operatorname{stab}\left(v_{+}\right)$to get a representation $\rho_{+}$of $\operatorname{stab}\left(v_{+}\right)$and $A_{i} \in \mathrm{SL}_{2}(\mathbf{C})$ such that

$$
A_{i} \cdot\left(\rho_{n_{i}} \mid \operatorname{stab}\left(v_{+}\right)\right) \cdot A_{i}^{-1} \rightarrow \rho_{+} .
$$


Apply Corollary 2.1 to the sequence of representations $\rho_{n_{i}} \mid \operatorname{stab}\left(v_{-}\right)$to get a representation $\rho_{-}$of $\operatorname{stab}\left(v_{-}\right)$and $B_{j} \in \mathrm{SL}_{2}(C)$ such that

$$
B_{j} \cdot\left(\rho_{n_{i_{j}}} \mid \operatorname{stab}\left(v_{-}\right)\right) \cdot B_{j}^{-1} \longrightarrow \rho_{-} .
$$

If every element $\gamma \in \operatorname{stab}(e)$ has $\operatorname{tr}\left(\rho_{+} \gamma\right)= \pm 2$ then Lemma 2.2 gives the result.

So we may assume there is an element $\gamma$ in $\operatorname{stab}(e)$ with $\operatorname{trace}\left(\rho_{+} \gamma\right)=c \neq$ \pm 2 . We claim that there is an element $\alpha_{+} \in \operatorname{stab}\left(v_{+}\right)-\operatorname{stab}(e)$ with the property that $\operatorname{trace}\left(\rho_{+} \alpha_{+}\right)=a \neq \pm 2$. Choose an element $\alpha \in \operatorname{stab}\left(v_{+}\right)-\operatorname{stab}(e)$. Note that $\alpha, \alpha \gamma, \alpha \gamma^{-1} \in \operatorname{stab}\left(v_{+}\right)-\operatorname{stab}(e)$ so that if any of these elements have $\operatorname{trace}(\rho) \neq \pm 2$ we are done. Otherwise set $A=\rho_{+} \alpha$ and $C=\rho_{+} \gamma$; then from

$$
\operatorname{tr}(A C)+\operatorname{tr}\left(A C^{-1}\right)=\operatorname{tr}(A) \operatorname{tr}(C)
$$

we see that $\pm 2 \operatorname{tr}(C)= \pm 2 \pm 2$, but $\operatorname{tr}(C)=c \neq \pm 2$ hence $\operatorname{tr}(C)=0$. Now

$$
\operatorname{tr}\left(A^{2} C\right)+\operatorname{tr}(C)=\operatorname{tr}(A) \operatorname{tr}(A C),
$$

thus $\operatorname{tr}\left(A^{2} C\right)= \pm 4$. If $\alpha^{2} \gamma$ is not in $\operatorname{stab}(e)$ we are done. Otherwise we can replace $\gamma$ in the above argument by $\alpha^{2} \gamma$ to conclude that $\alpha^{3} \gamma$ will do.

Similarly, we can assume there is $\alpha_{-} \in \operatorname{stab}\left(v_{+}\right)-\operatorname{stab}(e)$ with $\operatorname{tr}\left(\rho_{-} \alpha_{-}\right) \neq$ \pm 2 .

Consider the action of $\alpha_{+}$and $\alpha_{-}$on the tree $\Gamma$. These elements stabilize different vertices of $\Gamma$ and do not stabilize the edge between them, so the element $\alpha_{+} \cdot \alpha_{-}$acts as a non-trivial hyperbolic translation on $\Gamma$ (see [4]). Thus $\operatorname{tr}\left(\rho_{n}\left(\alpha_{+} . \alpha_{-}\right)\right) \rightarrow \infty$ as $n \rightarrow \infty$. It follows that the non-parabolic elements $\rho_{n}\left(\alpha_{+}\right)$and $\rho_{n}\left(\alpha_{-}\right)$have axes which are moving away from each other in $\mathbf{H}^{3}$. By a sequence of conjugacies, we may arrange that $\operatorname{Fix}\left(\rho_{n}\left(\alpha_{+}\right)\right)$is converging to 0 and $\operatorname{Fix}\left(\rho_{n}\left(\alpha_{-}\right)\right)$is converging to $\infty$.

Now consider any element $\beta \in \operatorname{stab}(e)$. Then $\beta \alpha_{ \pm} \beta^{-1} \in \operatorname{stab}\left(v_{ \pm}\right)$since $\operatorname{stab}(e) \subset \operatorname{stab}\left(v_{ \pm}\right)$. This implies that the axes of $\rho_{n}\left(\beta \alpha_{+} \beta^{-1}\right)$ and $\rho_{n}\left(\alpha_{+}\right)$ remain within a bounded distance of each other, since they are elements with trace bounded away from \pm 2 and their product is in $\operatorname{stab}\left(v_{+}\right)$and so has bounded trace. Similarly for the minus sign. It follows that for $n$ large, $\rho_{n}(\beta)$ moves 0 and $\infty$ by a very small amount. Thus $\rho_{n}(\beta)$ converges to a diagonal matrix as $n \rightarrow \infty$.

Suppose that $M$ is a connected 3-manifold and $F$ is a surface properly embedded, but possibly not connected, in $M$. We do not assume that either $M$ or 
$F$ is orientable; we do not assume that $F$ is incompressible. We show how to construct an action of $\pi_{1}(M)$ on a tree from this data.

Let $F_{1}, F_{2}, \cdots, F_{n}$ be the components of $F$ and $M_{1}, M_{2}, \cdots, M_{m}$ be components of $M-F$. Let $\pi: \tilde{M} \longrightarrow M$ be the universal cover of $M$. We construct a graph $\Gamma$ by assigning one vertex to each component of $\pi^{-1}(M-F)$ and one edge to each component of $\pi^{-1}(F)$. The edge corresponding to the component $\tilde{F}_{i}$ of $\pi^{-1}\left(F_{i}\right)$ is incident to the vertex corresponding to the component $\tilde{M}_{j}$ of $\pi^{-1}\left(M_{j}\right)$ if the closure of $\tilde{M}_{j}$ contains $\tilde{F}_{i}$. We must show that every edge is incident to precisely 2 vertices. To see this, note that there are either one or two components of $M-F$ adjacent to $F_{i}$. If there are two components, the result is clear. If there is only one component of $M-F$ adjacent to $F_{i}$, say $M_{j}$, then there is a loop in $M_{j} \cup F_{i}$ which meets $F_{i}$ once transversely. Thus this loop is essential, and hence in $\tilde{M}$ there are two distinct components of $\pi^{-1}\left(M_{j}\right)$ whose closure contains $\tilde{F}_{i}$. It is clear that the action of $\pi_{1}(M)$ on $\tilde{M}$ by covering transformations induces a simplicial action on $\Gamma$.

Next we show that $\Gamma$ is a tree. There is an embedding $i: \Gamma \longrightarrow \tilde{M}$ such that the image of each vertex of $\Gamma$ lies in the component of $\pi^{-1}(M-F)$ to which it corresponds, and so that the image of each edge of $\Gamma$ intersects once transversely $\pi^{-1} F$ in the component to which it corresponds. Observe that there is a neighborhood of $\tilde{F}_{i}$ in $\tilde{M}$ which is a product $I \times \tilde{F}_{i}$. This is because $\tilde{F}_{i}$ is properly embedded in $\tilde{M}$, and if $\tilde{F}_{i}$ is one-sided in $\tilde{M}$ then there is a loop in $\tilde{M}$ which meets $\tilde{F}_{i}$ once transversely, which implies that this loop is non-zero in $H_{1}\left(\tilde{M} ; \mathbf{Z}_{2}\right)$. However $\tilde{M}$ is simply connected, giving a contradiction. Therefore $\tilde{F}_{i}$ is 2 -sided in $\tilde{M}$. There is a retraction $r: \tilde{M} \longrightarrow i(\Gamma)$ defined by sending a product neighborhood $I \times \tilde{F}_{i}$ of $\tilde{F}_{i}$ onto the edge to which it corresponds by projection onto the $I$ factor, and sending a component of $\pi^{-1}(M-F)$ with these product neighborhoods removed to the vertex of $i(\Gamma)$ to which it corresponds. Since $\tilde{M}$ is simply connected, it follows that $i(\Gamma)$ is simply connected.

Now suppose that the boundary of $M$ contains an incompressible torus $T$ and that some component $S$ of $F$ meets $T$ in an essential loop $\alpha$. We now assume that $F$ is incompressible and contains no boundary parallel disc. The incompressibility of $F$ means that every component of $S \cap T$ is essential in $T$, and therefore parallel to $\alpha$. Let $\tilde{T}$ be a component of $\pi^{-1} T$. This implies that each $\pi_{1}\left(M_{i}\right)$ injects into $\pi_{1}(M)$, and thus $\tilde{M}_{i}$ is simply connected. It follows that $\tilde{M}_{i}$ meets $\tilde{T}$ in a connected, but possibly empty, set.

We choose a base point $\tilde{x} \in \tilde{T}$ and set $x=\pi(\tilde{x})$ in order to identify $\pi_{1}(M, x)$ with the covering transformations of $\tilde{M}$; then $\tilde{T}$ is stabilized by $\pi_{1}(T, x)$. Let $C_{1}, C_{2}, \cdots, C_{n}$ be the components of $T \cap S$ which are all parallel to $\alpha$, labelled 
in the order they go round $T$. The components of $\pi^{-1}\left(C_{1} \cup \cdots \cup C_{n}\right)$ are parallel lines on $\tilde{T}$. It follows that each component of $\tilde{T}-\pi^{-1}\left(C_{1} \cup \cdots \cup C_{n}\right)$ meets a distinct component $\tilde{M}_{j}$ of $\tilde{M}-\tilde{F}$ and thus corresponds to a distinct vertex in $\Gamma$. Thus the image of $\tilde{T}$ under $r$ is a line $\ell$ in $\Gamma$, and $r: \tilde{T} \longrightarrow \ell$ can be chosen to be a submersion.

We now assume that $S$ can be transversely oriented, that is, $S$ is 2-sided in $M$. Choose two arcs, one in $S$ and the other in $T-S$, from $C_{i}$ to $C_{i+1}$ with the same end points. The union of these two arcs is a loop $\gamma_{i}$. Push $\gamma_{i}$ off $S$ using the transverse orientation. We now assume that $[S]=0 \in H_{2}\left(M, \partial M ; Z_{2}\right)$. From this it follows that when the loop $\gamma_{i}$ is pushed off $S$ it must intersect $S$ an even number of times. This implies that if $C_{i}$ is isotoped along $T$ to $C_{i+1}$ then the transverse orientations of $S$ along $C_{i}$ and $C_{i+1}$ are opposite.

Each line $\tilde{C}_{i}$ in $\tilde{T} \cap \pi^{-1} S$ lies in a component of $\pi^{-1}(S)$, thus the edges of $\Gamma$ corresponding to the family of lines $\tilde{T} \cap \pi^{-1} S$ are in the same orbit under $\pi_{1}(M, x)$. Given a pair of adjacent lines $\tilde{C}_{i}, \tilde{C}_{i+1}$ in $\tilde{T} \cap \pi^{-1}\left(C_{i} \cup C_{i+1}\right)$, let $\boldsymbol{e}_{i}, \boldsymbol{e}_{i+1}$ be the corresponding edges in $\Gamma$. Orient $\ell$ and use this to orient each edge on $\ell$. Let $\tau: \pi_{1}(M, x) \longrightarrow \operatorname{Aut}(\Gamma)$ be the action of $\pi_{1}(M, x)$ on $\Gamma$. We will write $\tau_{\gamma}$ for $\tau(\gamma)$. Then for some $\delta_{i} \in \pi_{1}(M, x)$,

$$
\tau_{\delta_{i}}\left(e_{i}\right)=-e_{i+1},
$$

where the minus sign means with orientation reversed. This follows from the discussion of transverse orientations of surfaces above because an orientation of an edge $e_{i}$ corresponds to a transverse orientation of the corresponding surface $\tilde{S}$. We remark for later use that $\delta_{i}$ is in the free homotopy class of the loop $\gamma_{i}$ constructed above.

Now suppose that

$$
\tau^{\prime}: \pi_{1}(M, x) \longrightarrow \operatorname{Aut}\left(\Gamma^{\prime}\right)
$$

is a simplicial action without inversions on a simplicial tree $\Gamma^{\prime}$. Then there is an equivariant map

$$
f: \tilde{M} \longrightarrow \Gamma^{\prime}
$$

which is transverse to the midpoints of all edges of $\Gamma^{\prime}$, and this map may be chosen so that every component of the pre-image under $f$ of the midpoints of the edges of $\Gamma^{\prime}$ is an incompressible 2-sided surface $\tilde{F}$ in $\tilde{M}$. This surface $\tilde{F}$ in $\tilde{M}$ projects to a 2 -sided incompressible surface $F$ in $M$. (The condition that the action is without edge inversions is equivalent to $F$ being 2-sided.) We may 
apply the construction above to $F$ to get an action

$$
\tau: \pi_{1}(M, x) \longrightarrow \operatorname{Aut}(\Gamma)
$$

on a tree $\Gamma$. Clearly the map $f$ factors as $f=\bar{f} \circ r$, where $\bar{f}: \Gamma \longrightarrow \Gamma^{\prime}$ is an equivariant map.

Suppose now that $\pi_{1}(T, x)$ stabilizes no vertex of $\Gamma^{\prime}$. There is a line $\ell^{\prime}$ in $\Gamma^{\prime}$ which is stabilized by $\pi_{1}(T, x)$. We claim that $f$ may be chosen so that $\bar{f} \mid \ell$ is injective. $\tilde{T}$ is a plane on which $\pi_{1}(T, x)$ acts freely with quotient the torus $T . f \mid \tilde{T}$ covers a map $T \longrightarrow \ell^{\prime} / \pi_{1}(T, x)=S^{1}$, which is homotopic to a submersion. Lifting this homotopy gives a $\pi_{1}(T, x)$-equivariant homotopy of $f \mid \tilde{T}$. This can be used to give a homotopy of $f$ on all of $\tilde{M}$ by using a small collar neighborhood of $\tilde{T}$. This homotopy may then be done equivariantly to each component of $\pi^{-1}(T)$.

Let $e_{i}^{\prime}=f\left(e_{i}\right)$ and $e_{i+1}^{\prime}=f\left(e_{i+1}\right)$, then since $f \mid \ell \longrightarrow \ell^{\prime}$ is an an equivariant simplicial homeomorphism it follows from equation (1) that

$$
\tau_{\delta_{i}}^{\prime}\left(e_{i}^{\prime}\right)=-e_{i+1}^{\prime} .
$$

Let $S$ be a component surface of $F$ which we assume is oriented. Use this orientation to orient the boundary components $C_{1}, C_{2}, \cdots, C_{n}$ of $S$. The base point $x$ is chosen on $C_{1}$, and let $c_{1}, c_{2}, \cdots, c_{n}$ be elements of $\pi_{1}(S, x)$ which correspond to the oriented boundary components of $S$. Thus $c_{1}, c_{2}, \cdots, c_{n}$ is a commutator in $\pi_{1}(S, x)$. Since $C_{i}$ and $C_{i+1}^{-1}$ are isotopic on $T$, the elements $c_{i}$ and $c_{i+1}^{-1}$ are conjugate so there is an element $\delta_{i} \in \pi_{1}(M, x)$ with $\delta_{i} \cdot c_{i} \cdot \delta_{i}^{-1}=c_{i+1}^{-1}$. Clearly the covering transformation of $\tilde{M}$ corresponding to $\delta_{i}$ sends $\tilde{S}_{i}$ to $\tilde{S}_{i+1}$ and thus $\delta_{i}$ satisfies (1) and hence (2).

DEFINITION. Suppose that $\pi_{1}(M)$ acts on a tree, then a surface $F$ in $M$ is called a reduced surface associated to the action if it is associated to the action and has the minimal number of boundary components.

We can now give a proof of Theorem 1.1.

PROOF OF THEOREM 1.1. Let $S$ be a component of a reduced surface associated to the limiting action on a tree. We continue to use the notation $c_{1}, c_{2}, \cdots, c_{n}$ used above for elements of $\pi_{1}(S, x)$ corresponding to the boundary components of $S$. Thus $\pi_{1}(S, x)$ is a subgroup of $\operatorname{stab}(e)$ for some edge $e$ of $\Gamma$. Let $\lambda$ be a limiting eigenvalue of $\rho_{n}(C)$.

If the surface $S$ does not separate, then $S$ must be a Seifert surface for the knot and have a single boundary component. The last sentence of this proof 
gives the result in this case. Thus we may assume that $S$ separates, and thus has an even number of boundary components. By Proposition 2.1 we may assume that $\rho_{m} \mid \operatorname{stab}(e)$ converges to a diagonal representation $\rho$ so:

$$
\rho\left(c_{i}\right)=\left(\begin{array}{cc}
\lambda & 0 \\
0 & \lambda^{-1}
\end{array}\right)^{\epsilon_{i}} \quad \text { for all } i
$$

where $\epsilon_{i}= \pm 1$. If $\lambda= \pm 1$ there is nothing to prove. Otherwise for $m$ large $\rho_{m}\left(c_{i}\right)$ has trace bounded away from \pm 2 and therefore the endpoints of the axis of $\rho_{m}\left(c_{i}\right)$ are converging to $0, \infty$. Now there is $\delta_{i} \in \pi_{1}(M)$ with $\delta_{i} \cdot c_{i} \cdot \delta_{i}^{-1}=c_{i+1}^{-1}$, and $\delta_{i}$ satisfies (2), hence $\rho_{m}\left(\delta_{i}\right)$ almost switches 0 and $\infty$. It follows that $\rho_{m}\left(c_{i}\right)$ and $\rho_{m}\left(c_{i+1}\right)$ are almost equal, and hence that all the $\epsilon_{i}$ are equal.

The homotopy class $\gamma=c_{1} . c_{2}, \cdots . c_{n}$ is a commutator; therefore by Proposition $2.1, \rho(\gamma)=I$, hence $\lambda^{n}=1$.

\section{References}

[1] M. Bestvina, 'Degenerations of the hyperbolic space', Duke J. Math. 56 (1988), 142-161.

[2] D. Cooper, 'Degenerations of representations in $\operatorname{SL}(2, C)$ ', preprint.

[3] M. Culler, D. Cooper, H. Gillett, D. D. Long and P. B. Shalen, 'Plane curves associated to character varieties of knot complements', preprint.

[4] M. Culler and J. Morgan, 'Group actions on R-trees', Proc. London Math. Soc. 55 (1987), $571-604$.

[5] M. Culler and P. B. Shalen, 'Varieties of group representations and splittings of 3-manifolds', Ann. of Math. 117 (1983), 109-145.

[6] A. Hatcher and W. P. Thurston, 'Incompressible surfaces in two bridge knot complements', Invent. Math. 79 (1985), 225-246.

Department of Mathematics

University of California

Santa Barbara, Ca. 93106

USA
Department of Mathematics

University of California

Santa Barbara, Ca. 93106

USA 